\title{
Protée
}

\section{La répétition esthétique}

\section{Yves Raymond}

Volume 27, numéro 1, 1999

\section{La Mort de Molière et des autres}

URI : https://id.erudit.org/iderudit/030547ar

DOI : https://doi.org/10.7202/030547ar

Aller au sommaire du numéro

\section{Éditeur(s)}

Département des arts et lettres - Université du Québec à Chicoutimi

\section{ISSN}

0300-3523 (imprimé)

1708-2307 (numérique)

Découvrir la revue

\section{Citer cet article}

Raymond, Y. (1999). La répétition esthétique. Protée, 27(1), 87-92.

https://doi.org/10.7202/030547ar

\section{Résumé de l'article}

Cet article propose un examen de la répétition esthétique en tant que processus de signification. En se fondant sur la notion peircienne d'interprétant et sur la notion wittgensteinienne de ressemblances de famille, l'auteur définit la répétition comme une action qui déclenche le processus de la pensée, de la recherche et de l'élaboration du sens. Trois fonctions caractéristiques de la répétition esthétique - la manifestation, la relation et la démultiplication - sont déterminées à travers la description d'une occurrence répétitive importante de la vidéo de Wilson. Cette brève analyse suggère non seulement que la répétition influence la réception de l'oeuvre par son pouvoir de manifestation, mais aussi que sa capacité relationnelle autorise l'élaboration d'un signe interprétant.
Ce document est protégé par la loi sur le droit d'auteur. L’utilisation des services d'Érudit (y compris la reproduction) est assujettie à sa politique d'utilisation que vous pouvez consulter en ligne.

https://apropos.erudit.org/fr/usagers/politique-dutilisation/ 


\section{LA RÉPÉTITION ESTHÉTIQUE}

YVES RAYMOND

L'utilisation par Wilson de la répétition esthétique dans ses productions n'est pas nouvelle. Déjà dans Le Regard du sourd et dans Prologue, deux productions théâtrales, Wilson utilisait la répétition comme moteur d'une représentation asystématique et non linguistique. Dans La Mort de Molière, l'opération répétitive est engagée dans plusieurs des éléments constituant la vidéo, qu'il s'agisse du texte (la narration), des images, des gestes des acteurs, de l'organisation des plans et des séquences, de la musique, du bruitage, etc. La vidéo comprend en effet de multiples éléments en série (bruits, cris, onomatopées, mots, gestes, personnages, actions, objets, lumières, couleurs, rythmes, images, mouvements de caméra, séquences, plans, etc.) et ces divers éléments établissent entre eux des relations basées sur des jeux de comparaisons, de ressemblances, de différences et d'analogies.

L'œuvre est perçue comme une diversité d'impressions manifestées par une série d'éléments chargés de significations ou d'un certain potentiel interprétatif. Cette situation, cependant, exige du spectateur un travail de réflexion, particulièrement pour celui qui veut arriver à un degré suffisant de compréhension et de satisfaction esthétique, de plaisir.

La réception étant fondée sur la mémoire, celle-ci est stimulée par les répétitions esthétiques, ce qui permet à la pensée d'entreprendre un processus de recherche, d'enquête, en se basant sur sa faculté de reconnaissance engendrée par le pouvoir de manifestation, de relation et de démultiplication de la répétition. L'espoir de mettre en lumière le fonctionnement de cette opération répétitive dans le contexte de l'œuvre vidéographique, et plus spécifiquement dans La Mort de Molière, est notre seul objectif ici.

\section{LA RÉPÉTITION ESTHÉTIQUE}

En périphérie du terme "répétition", on trouve ceux de réplique, de citation, d'échantillon, de rythme, de double, de mimétisme, d'emprunt, etc., mais la répétition est avant tout une action. Si on se place du point de vue de l'art, la répétition apparaît comme un acte de communication qui reproduit des 
représentations ou des signes déjà structurés dans une œuvre. La répétition, de ce point de vue, pourrait bien être un autre mécanisme sémiotique.

Il existe une idée de la répétition dans la définition de la notion centrale de la théorie de Peirce, celle d'interprétant. Les deux termes, signe et interprétant, apparaissent dans une définition assez connue du signe triadique, datant de 1897:

Un signe, ou representamen, est quelque chose qui tient lieu pour quelqu'un de quelque chose sous quelque rapport ou à quelque titre. Il s'adresse à quelqu'un, c'est-à-dire crée dans l'esprit de cette personne un signe équivalent ou peut-être un signe plus développé. Ce signe qu'il crée, je l'appelle l'interprétant du [...] signe. ${ }^{1}$ (C.P. 2.228; cf. 1978: 121)

La première caractéristique concerne l'idée de «lieutenance» du signe. Ce caractère essentiel est nommé le representamen et constitue la forme élémentaire de la relation sémiotique. L'interprétant est aussi considéré comme l'élément constitutif du fait que quelque chose est un signe. Il est un élément structural du signe. Celui-ci est créé, produit par l'interaction de trois éléments et cette interaction est ce que Peirce nomme la sémiose. Par «sémiose», il entend:

[...] une action ou influence qui est ou implique la coopération de trois sujets, tels qu'un signe, son objet et son interprétant, cette influence tri-relative n'étant en aucune façon réductible à des actions entre paires. $(5.484$; cf. 1978: 133)

La fonction principale du signe consisterait à influencer un esprit qui comprend sa méthode de signification, cette méthode étant sa substance signifiée ou l'interprétant. Ce serait donc grâce à cet interprétant créé dans notre esprit que nous serions conduit à reconnaître la relation entre l'objet et le signe qui le représente. Cette représentation sémiotique, comme le souligne Peirce, peut se réaliser soit en étant plus développée que le signe dont elle tient lieu, soit en étant équivalente à ce même signe. L'idée d'un interprétant plus développé suggère le caractère fondamental de la croissance de la signification, mais l'idée d'interprétant équivalent se rapproche peut-être de ce que nous croyons être des répétitions.

Dans la foulée de cet élan pragmatique, l'idée wittgensteinienne des « ressemblances de famille» ${ }^{2}$ pourrait aussi nous aider à comprendre le rôle de la répétition. Tentons de rappeler le sens et l'usage du mot «ressemblance» chez Wittgenstein. Un langage et une forme de vie ne possèdent pas de limites nettes, stables ou définitives. Pour Wittgenstein, les jeux de langage, les règles, les pratiques qui appartiennent à une forme de vie sont apparentés. Les jeux de langage et les formes de vie ne sont pas fermés sur eux-mêmes. Ils permettent au contraire de comprendre autrement et offrent la possibilité de faire des comparaisons, de présenter d'autres possibilités de compréhension. Les ressemblances de famille forment un réseau d'analogies qui s'entrecroisent et s'enveloppent les unes aux autres, analogies d'ensemble comme de détail. Wittgenstein s'attarde ainsi à clarifier une espèce de phénomènes à la fois complexes et familiers, dont la particularité est de ne pas entrer directement dans le champ d'application des règles et de la grammaire. Ces phénomènes, essentiellement liés à la vision, sont particuliers non seulement parce qu'ils semblent ne pas pouvoir trouver leur définition dans des règles, mais aussi parce qu'ils sont apparentés au genre de propriétés qu'on a coutume d'attribuer aux objets qui suscitent des réactions esthétiques ${ }^{3}$.

Quels rapports ces deux termes, sémiose et ressemblances de famille, ont-ils avec la répétition? Pour Peirce, la sémiose consiste en une détermination de plus en plus fine de ce qui est, et pour Wittgenstein, ce qui est se détermine progressivement dans l'établissement des relations de famille. Or, il semble bien que la répétition esthétique réponde à ces caractéristiques pragmatistes. Premièrement, la répétition s'inscrit nécessairement dans une série et cette série peut être infinie. En fait, toutes les significations doivent se ramener à des effets pratiques dans telle ou telle circonstance. L'usage, conséquemment, est quelque chose qui se concrétise non pas dans l'opinion, mais dans des opérations effectuées. La répétition pourrait apparaittre en ce sens 
comme un outil (esthétique), parce qu'elle offre des possibilités d'usage qui dépendent des fins visées, des usages antérieurs, d'un contexte d'activités et de ressources techniques diverses, ainsi que de certaines capacités qui sont elles-mêmes fonctions de conditions naturelles.

\section{LES MODALITÉS DE LA RÉPÉTITION}

La répétition dans son contexte vidéographique et plus généralement spectaculaire peut se manifester dans tous les éléments perceptibles qui constituent un élément signifiant audiovisuel. À la première étape de cette manifestation répétitive, un certain élément peut être reproduit sans être changé. L'élément fixe passe d'un contexte à un autre sans changer de forme. C'est le contexte qui, en changeant, transforme la fonction significative de l'élément fixe à travers la série. C'est ainsi que les séries de "Ce n'est pas un poème sur Molière» ou de «Molière se meurt» ou de «Father, I am dying» du discours narratif sont des éléments fixes que l'on retrouve dans différents contextes, c'est-à-dire ailleurs dans le temps et dans l'espace de l'œuvre. La série sonore des «cris», du «verre brisé» ou des «hum» en constitue d'autres exemples. Mais il y a dans $L a$ Mort de Molière un exemple particulièrement frappant de ce processus de la répétition: cet élément visuel simple, le «fauteuil», qui, se trouvant répété de manière systématique, donne prise à un processus particulier de perception, d'interprétation et de compréhension.

À travers les dix séquences de la vidéo, on trouve dix-neuf manifestations du «fauteuil». Examinons-en les différentes apparitions. Dans la première séquence, il apparaît pour la première fois lorsque, au plan 5 , un homme dépose un fauteuil en cuir. Dans la deuxième séquence, le même fauteuil se présente cette fois en plongée, trois quarts de face (plan 12). Toujours dans cette séquence, au plan 17, le fauteuil est vu de dos comme à la fin du plan 5 . À la séquence $2 \mathrm{~b}$, plan 19 , le fauteuil apparaît comme au plan 12 , de même qu'au début de la troisième séquence, plan 22, et encore au début de la séquence 4 , plan 26 . Au plan 34 , toujours dans la quatrième séquence, le docteur s'arrête devant le fauteuil et frappe le dossier avec le poing. Au début de la cinquième séquence, plan 36, le fauteuil est présenté comme au plan 12. Le plan 38 de la cinquième séquence est un travelling ascendant qui débute par le bas du fauteuil, passe par des jambes, monte jusqu'à des mains qui trempent un torchon dans un pot et qui nettoient nerveusement le fauteuil. L'image montre ensuite le visage du père de Molière, qui cire nerveusement l'accoudoir du fauteuil. Puis, plan 41 , on voit en plan rapproché le père derrière le fauteuil; perturbé et absent, il s'éloigne vers la gauche. La séquence s'achève avec l'image du dossier du fauteuil: un travelling arrière et descendant nous le fait découvrir seul dans un carré blanc. Au début de la sixième séquence, plan 42 , le fauteuil apparaît comme au plan 12. Au plan 44, des pieds d'enfant nus vont jusqu'au carré blanc. L'enfant s'assoit dans le fauteuil, travelling ascendant jusqu'à l'enfant qui fait une grimace. À l'arrière-plan, on voit Molière dans son lit. Au plan 48 de la même séquence, le fauteuil apparaît de face avec, à l'arrière-plan, le docteur qui s'approche comme s'il s'interrogeait: il se gratte la tête, puis s'éloigne vers la droite. Au début des septième et huitième séquences, c'est-à-dire plans 55 et 59 , le fauteuil apparaît comme au plan 12. À la fin de la huitième séquence, plan 61, le fauteuil est sous verre, dans le carré blanc, et entouré de gens assis sur des chaises. Dans la dixième et dernière séquence, le fauteuil est d'abord présenté comme au plan 12 et il réapparaît une dernière fois, plan 89 , de face.

À ce premier niveau de manifestation, la répétition s'impose à nous en tant que possibilité vague, sans temporalité ou spatialité déterminée, de l'établissement d'une relation entre le signe (representamen) et ce dont il tient lieu. Mais la répétition semble convertible, c'est-à-dire perçue à la fois dans ses diverses manifestations et dans ses relations avec d'autres éléments. Les différentes formes de transmission d'un élément donnent aussi naissance à un complexe d'éléments plus riches.

Voyons d'abord le rapport de la série avec la temporalité. La répartition, dans le temps chronologique de la vidéo, des répliques ou des 
occurrences de la série du fauteuil révèlent une rythmique particulière, une sorte de ponctuation de l'œuvre. Chaque séquence comporte au moins une apparition de l'élément fixe. Sauf pour la première séquence, où le fauteuil est montré au plan 5 , toutes les autres séquences débutent par l'image du fauteuil. Le retour régulier de cette image permet de percevoir une progression dans le temps de la fiction en établissant une liaison entre chacune des séquences. Sur un plan spécifiquement formel, la répétition du fauteuil permet d'identifier une structure organisatrice.

Cependant, ce n'est pas là la seule relation possible. Par exemple, les images du fauteuil apparaissant aux plans 5, 12, 19, 22, 26, 55, 59 et 62 sont accompagnées de la phrase "Molière se meurt» et celle du plan 74 de la phrase «Molière est mort». Cette relation texte-image offre une régularité qui permet de déterminer un élément temporel important: la fin. Elle permet également d'établir une relation entre le fauteuil et Molière. Par ailleurs, d'autres occurrences de l'image du fauteuil apparaissent dans l'action même de la fiction. Il y a six manifestations de ce type que l'on retrouve aux plans $34,38,41,44,48$ et 61 , décrits ci-dessus. On frappe sur le fauteuil, on le nettoie, on s'y assoit pour faire une grimace, on se questionne sur lui, on l'expose.

Si on examine maintenant la série dans sa relation à l'espace, on s'aperçoit par exemple que les plans 12 , $19,22,26,36,42,55,59,62$ et 74 , où l'on voit le fauteuil sous verre, en plongée et trois quarts de face, comportent exactement les mêmes caractéristiques spatiales. La première de celles-ci est certainement la décontextualisation de l'espace. Le fauteuil sous verre apparait quelque part certes, mais ce quelque part n'est pas déterminé. Par ailleurs, ces plans se distinguent quant à leur durée et sont accompagnés de textes, de musiques et de bruits différents. Pour les plans 34,38 , $41,44,48$ et 61 , où le fauteuil est intégré à l'action, on constate une plus grande contextualisation. Les lieux sont davantage déterminés, car ils contiennent des objets, des personnages, etc. Enfin, pour les plans 5, 17, 61 et 89, dans lesquels le fauteuil est présenté de dos ou de face, la contextualisation de l'espace encore une fois n'existe pas. D'ailleurs la rapidité de leur présentation fait qu'il est difficile lors d'une première réception de les percevoir autrement que de façon subliminale.

On ne voit déjà plus, ici, la série comme une simple possibilité, mais comme une manifestation de faits particuliers, déterminés par leur opposition à tout le reste et par le fait qu'ils sont composés, c'est-àdire constitués d'éléments distincts possédant des connexions particulières. Il s'agit ici du mode dyadique où les connexions se manifestent en une multitude de relations entre deux éléments. C'est la démultiplication des éléments fixes et leur propriété de relations complexes qui permettent à la pensée d'établir certaines associations qui sont fondées sur les modes de représentation de l'objet. Comment par exemple peut-on arriver à associer l'image du fauteuil avec celle de Molière? En d'autres termes, quelles sont les ressemblances qui font que nous pouvons comparer ou associer les deux éléments?

Nous arrivons ici au problème de l'interprétant. L'interprétant est ce par quoi quelque chose est signe. Il y a trois types d'interprétant. L'interprétant immédiat est produit par le signe lui-même sans tenir compte de son contexte ni des circonstances de sa production. Il survient avant toute réflexion, comme une impression. Cette impression peut être diverse: elle peut être une possibilité qualitative, une information attribuable à un objet ou une régularité. L'interprétant immédiat est en quelque sorte une hypothèse fondée sur certaines impressions non déterminées. À ce premier stade, nous serions porté à suggérer que le fauteuil est un signe représentant Molière. L'interprétant dynamique, pour sa part, désigne l'effet que le signe produit. Il est de l'ordre du fait, de la réalisation. L'interprétant dynamique peut être émotionnel (ou affectif), énergétique (impliquant un effort le plus souvent mental) ou logique (impliquant une règle générale, une habitude d'action). Mais la règle ou l'habitude qu'il exprime sont incarnées dans un ensemble fini d'actions et selon une durée limitée. Vient ensuite l'interprétant 
final (ou normal), qui est une habitude vivante, c'est-àdire active, qui peut encore se perfectionner. L'interprétant final ne peut pas limiter de façon définitive l'engendrement des interprétants. Il est ce qui survient après un développement suffisant de la pensée. Le terme est alors atteint en conformité avec un but visé.

Dans cette élaboration de la série de répétitions esthétiques manifestée par les occurrences des images du fauteuil, y a-t-il une sémiose qui opère? Pouvonsnous remplacer le «fauteuil» par «Molière»? Il est clair que le fauteuil représente Molière et sa représentation est selon les cas métaphorique, métonymique ou analogique. Les images du fauteuil sont d'abord perçues comme une régularité et cette régularité excite notre imagination et nous engage à trouver une signification. Toutefois, celle-ci ne peut s'établir que par le truchement de la manifestation, de la mise en relation et la démultiplication. La répétition de l'image du fauteuil permet à la mémoire de retenir, d'abord de façon assez vague, la manifestation d'une régularité qui, à son tour, déclenche le processus de la pensée, processus qui permet d'élaborer un signe interprétant.

\section{Notes}

1. Toutes les citations de Peirce, directes ou indirectes, renvoient aux Collected Papers. Selon l'usage habituel, ces extraits sont référencés par volume et paragraphe.

2. On peut trouver un écho de la discussion sur les « ressemblances de famille» notamment dans les Investigations philosophiques, $§ 65$ à 68, où Wittgenstein examine les «jeux de langage", ainsi qu'aux $\$ 138$ à 142, où il est question des règles et de la compréhension de la série.

3. Cette discussion a lieu principalement dans Les Leçons et conversations sur l'esthétique, mais aussi au chap. XI de la $2^{\mathrm{e}}$ partie des Investigations philosophiques.

\section{RÉFÉRENCES BIBLIOGRAPHIQUES}

PeIRCE, C.S. [1931-1935]: Collected Papers, vol. I à VI (sous la dir. de C. Hartshorne et P. Weiss), Harvard, Harvard University Press; [1958]: Collected Papers, vol. VII et VIII (sous la dir. de W. Burks), Harvard, Harvard University Press;

[1978] : Écrits sur le signe (rassemblés, traduits et commentés par G. Deledalle), Paris, Éd. du Seuil.

WiTTGENSTEIN, L. [1971]: Leçons et conversations sur l'esthétique, la psychologie et la croyance religieuse, Paris, Gallimard (pour la trad. française);

[1961]: Tratatus logico-philosophicus suivi de Investigations philosophiques, trad. de l'allemand par P. Klossowski, Paris, Gallimard, coll.

"Bibliothèque des Idées". 


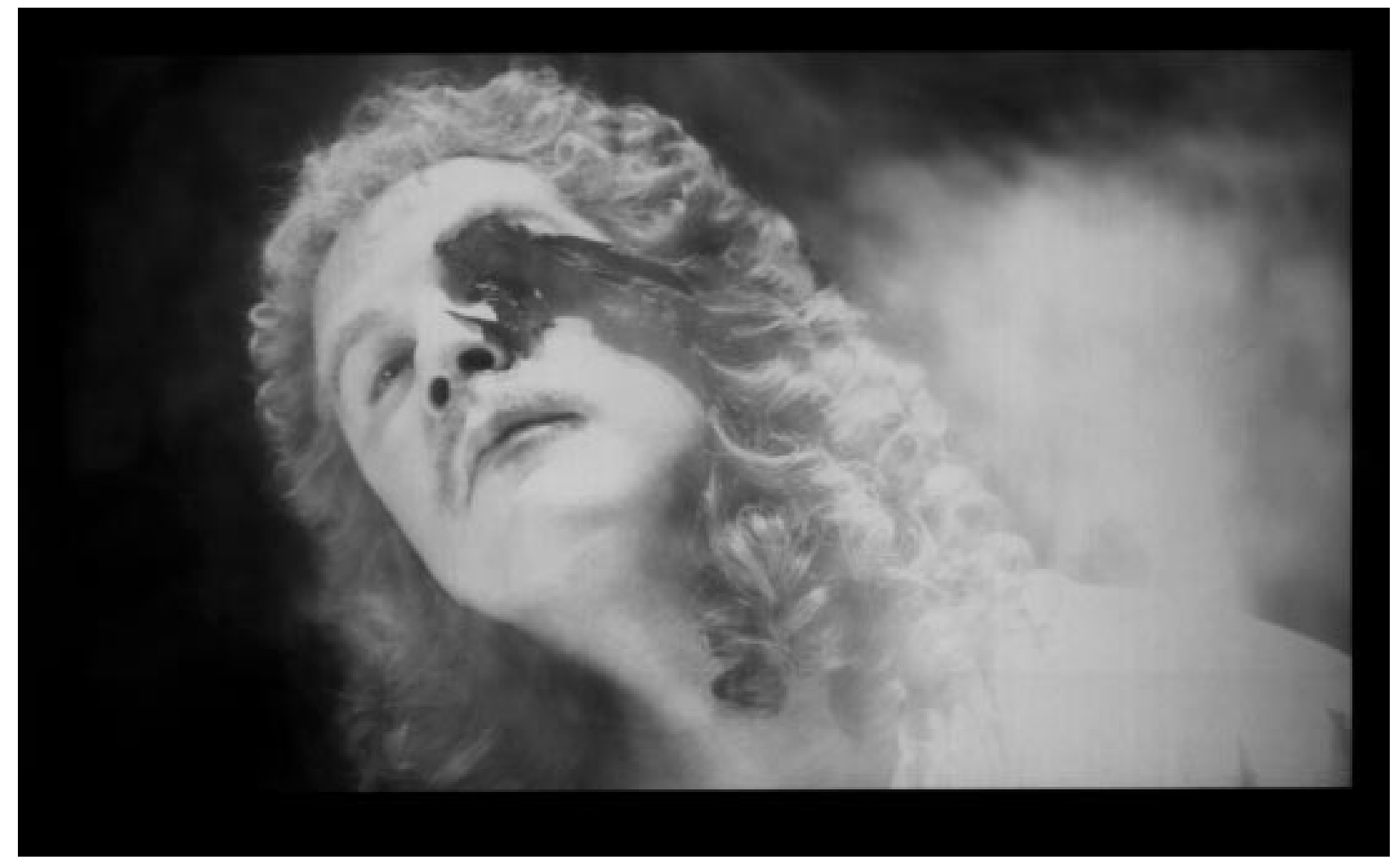

La Mort de Molière de Robert Wilson. Photo INA. 